\title{
Shape-shifting of polymer beams and shells due to oil extraction
}

\author{
Daniele Battista $^{\mathrm{a}}$, Valeriy Luchnikov ${ }^{\mathrm{b}}$, Paola Nardinocchi ${ }^{\mathrm{a}, *}$ \\ ${ }^{a}$ Dipartimento di Ingegneria Strutturale e Geotecnica \\ Sapienza Università di Roma, I-00184 Roma, Italy \\ ${ }^{b}$ Institut de Science des Matériaux de Mulhouse \\ 15 rue Jean Starcky Mulhouse, FR 68057
}

\begin{abstract}
We investigate the morphing of bilayer naturally curved beams and cylinders due to oil extraction from the outer layers. We fabricate bilayer naturally curved beams and cylindrical shells made of PDMS/(PDMS + silicone oil), use the experimental results to validate an explicit formula delivering the change in curvature of the beams, based on the modeling of oil extraction as a bulk contraction. We show as the same model, set up within a $3 \mathrm{D}$ context, delivers the morphing of bilayer cylinders and identify potentially interesting results for designing future experiments. In particular, we show as stable states corresponding to the original cylinders can be got in the form of saddle-like shells and cylinders with the axes of principal curvatures switched with respect to the original axes.
\end{abstract}

Keywords: Shape-morphing, Bilayer beams, Cylindrical shells, Oil extraction

\section{Introduction}

In the last decades, polydimethylsiloxane (PDMS) has become popular for the rapid prototyping of many devices widely used in manifacturing and biomedical industries [1]. This is due to the many advantageous characteristics of the material: transparency, biocompatibility, flexibility, high cost-effectiveness and good moldability. 22 PDMS is a rubber-like material. However, owing to its porous nature and compatibility with organic solvents [3], it shows a gel-like behaviour swelling when a solvent diffuses into the polymer and, viceversa, shrinking when it comes out from it. This property, together with the PDMS hydrophobicity, allowed to realize several devices: sponges for water-oil separation [4, 5, 6], solvent-tunable microlens [7], wrinkled microelectrodes [8], and wireless passive radio frequency identification sensors (RFID) based on PDMS swelling caused by organic vapour solvents 9. In functionalized or composite PDMS, deformation can also be

${ }^{*}$ Corresponding Author: P. Nardinocchi, Dipartimento di Ingegneria Strutturale e Geotecnica, via Eudossiana 18, I-00184 Roma, tel: 003906 44585242, fax: 0039064884852.

Email addresses: daniele.battista@uniroma1.it (Daniele Battista), valeriy.luchnikov@uha.fr (Valeriy Luchnikov), paola.nardinocchi@uniroma1.it (Paola Nardinocchi) 
induced in response to external stimuli as $\mathrm{pH}$ [10], UV irradiation [11] and magnetic field [12].

Some drawbacks may arise when the swelling(shrinking)-induced deformation of PDMS structures is undesired. For example, the modification of PDMS microchannel geometries due to swelling can affect the trajectories of droplets [13. In some cases, such as after the extraction of silicone uncrosslinked chains [14] or after ion beam and electron beam irradiation [15] these undesired deformations are irreversible, too.

In the present paper, we investigate shape-morphing of PDMS-based thin bilayer structures, which is a quite common configuration employing PDMS as both passive [16] and active layer [17] (see also [18]). Mismatch strains due to expansion(shrinkage) of the active layer can induce a macroscopic bending or surface instabilities, depending on different material and geometrical parameters [19. Slender bilayer self-bending soft structures can be used as sensors and actuators in soft robotics, as drug delivery system, or can represent the single element of an architectural material. All these situations require an accurate control of the deformation process and we wish to make some progress towards addressing this question.

We present the results of a campaign of experimental tests made on bilayer naturally curved PDMS beams, with the outer layer containing oil. The beams are made to flatten and evert through oil extraction from the outer layer. The final change in curvature of the beam can be explicitly determined starting from the spontaneous deformation shrinking deformation of the outer layer due to oil extraction. The explicit model is borrowed from [20] and it is shown to be in good agreement with the experimental results. Shape-shifting due to oil extraction in bilayer PDMS cylindrical shells is also presented in terms of a model set within the context of finite elasticity with spontaneous deformations. The model is solved via finite element method and it allows to determine the amount of oil to put in the outer layer of a cylindrical shell of given axis and curvature to get after oil extraction a cylinder with axis orthogonal to the original one, saddle-like shapes and double-curved shapes.

\section{Material and methods}

The goal of our experiments is inducing bending in bilayer naturally curved PDMS beams through shrinking of just one of the two layers. The naturally curved beams are obtained by cutting them from a cylindrical bilayer thin structure in the hoop direction just after the end of the curing process described in the following. The preparation of the bilayer cylinders is resumed in the cartoon in figure 1. The procedure followed to realize the two layers had already been used by one of the Authors and exhaustively described in 21. Shortly, one layer is made up of polydimethylsiloxane (PDMS) (SYLGARD ${ }^{\text {TM }}$ 184 ) at a $10: 1$ ratio of prepolymer/crosslinker (the passive layer). The second (active) layer is made up of PDMS mixed with silicone oil (Rhodorsil ${ }^{\circledR}$ Oil 47V20) at $10: x: 1$ ratio of prepolymer/oil/crosslinker, with $x=2,4,6,8,10$; during the curing process, as the PDMS reticulates, the silicone oil remains trapped into the polymer matrix. 
The external (active) layer of the cylindrical shell is formed by pouring the PDMSoil-curing agent liquid mixture into the space between the hollow cylinder (B) of internal radius $22 \mathrm{~mm}$ and the full cylinder $(\mathrm{C})$ of radius $20 \mathrm{~mm}$. After degassing and curing at $100^{\circ} \mathrm{C}$ for 1 hour, the cylinder (C) is extracted and another one (D) of smaller radius $18 \mathrm{~mm}$ is inserted coaxially to (B). The inner (passive) layer is formed by pouring the PDMS-curing agent liquid mixture into the space between the outer layer just crosslinked and the cylinder (D). It follows a second degassing and heating, at $100^{\circ} \mathrm{C}$ for a time sufficient to complete all chemical reactions (about 2 hours). After the mold opening the two parts result glued each other and form a cylindrical bilayer shell with middle radius $20 \mathrm{~mm}$ and thickness $4 \mathrm{~mm}$. Already at this stage, it is expected that the oil starts partially diffusing through the inner layer. However, we do not observe relevant effects on the curving of the sample since the curing time is much shorter than the diffusion time.

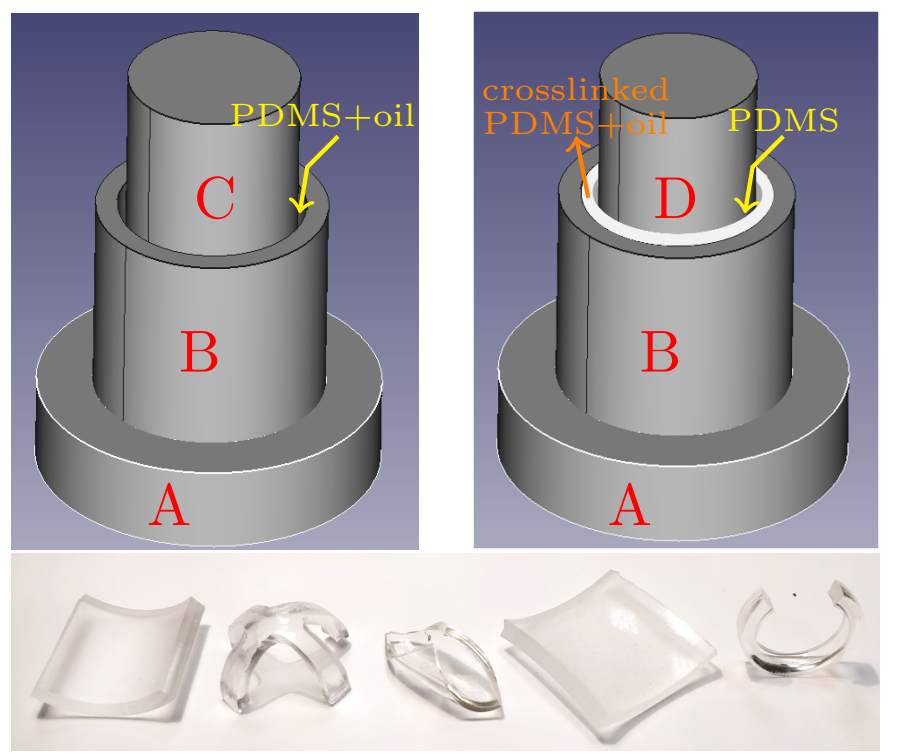

Figure 1: Top: bilayer cylinder fabrication. The outer layer is formed by pouring the PDMS-oil-curing agent liquid mixture into the space between the hollow cylinder (B) and the full cylinder (C). After degassing and curing, the cylinder (C) is removed and (D) is inserted coaxially to (B). The inner layer is formed by pouring the PDMS-curing agent liquid mixture into the space between the outer layer just crosslinked and the cylinder (D). Bottom: some shapes made by the same bilayer structure fabricated by using the same kind of procedure.

The samples are then immersed in a toluene bath. The complete extraction of the oil is produced in a few hours. The final configuration which the bilayer beam gets is bent with respect to the initial, stable and maintained. 


\section{Beam flattening and eversion induced by oil extraction}

In the process described above, four main phases can be identified: i) diffusion of oil from the outer (active) to the inner (passive) layer, which takes place even when the layers are still in the mold; ii) swelling of the bilayer in toluene; iii) diffusion of oil from the swollen bilayer into the toluene bath; iv) evaporation of toluene. At the stationary state, corresponding to the complete oil extraction, the PDMS bilayer beam looks like bent, due to the loss of oil from one layer, and swollen, due to the toluene absorption by both layers. The final shape is attained when all the toluene is evaporated and the bilayer is shrinked. Figure 2 shows the final shapes of a set of beams sharing the same initial beam curvature $\kappa_{c}$ and thickness $h$ but different one from another for the silicone oil content $x$ : the ratio of prepolymer/oil/crosslinker is $10: x: 1$ and $x=2,4,6,8,10$. It is shown that there are two values of $x$ which determine, after oil extraction, beam flattening $(x=6)$ and beam eversion $(x=10)$.

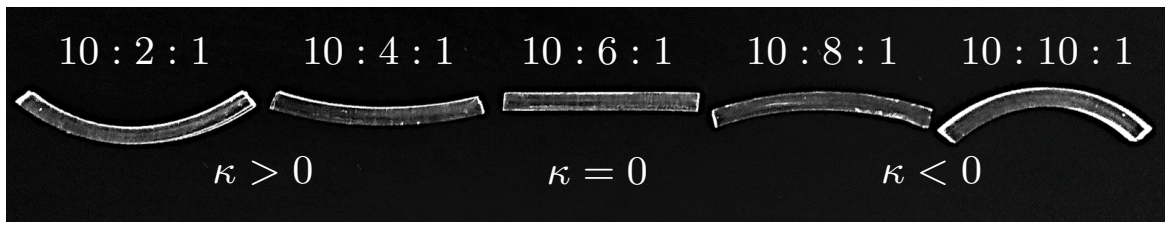

Figure 2: Bilayer naturally curved beams after oil extration for several values of the oil fraction. The initial configuration has a curvature $\kappa_{c}=50 \mathrm{~m}^{-1}$, thickness $h=4 \mathrm{~mm}$. The oil is in the outer layer.

As observed, oil extraction from the outer layer induce relevant deformations in the naturally curved beam, which produce beam flattening and eversion. In this situation, the well-known linear Timoshenko formula 22 fails in describing the change in curvature, as it fails when swelling-induced eversion and flattening in gel beams is studied [20, because it is valid for small deformations and slightly naturally curved beams. We adopt the explicit nonlinear formula presented in Ref. [20] to evaluate the curvature $\kappa$ acquired by the beam at the end of the extraction process. It has been shown to depend on geometrical parameters such as the beam thickness $h$, the layer thicknesses $h_{i}$ and $h_{e}$ (i=inner layer, e=external layer), and the initial curvature $\kappa_{c}$ as well as on the shear moduli $G_{i}$ and $G_{e}$ of the two layers, and on the natural longitudinal deformations $\lambda_{o i}$ and $\lambda_{o e}$. These latter are defined as the deformations which would take place in the two layers if they were free one from another. The curvature formula has the following form ${ }^{2}$

$$
\kappa=\hat{\kappa}\left(\alpha, \beta, \Gamma, \bar{\kappa}_{c}, h, \lambda_{o e}\right),
$$

with $\alpha=G_{i} / G_{e}, \beta=h_{i} / h, \Gamma=\lambda_{o e} / \lambda_{o i}$ and $\bar{\kappa}_{c}=\kappa_{c} h$.

\footnotetext{
${ }^{2}$ See the appendix of Ref. [20] for the explicit formula
} 




Figure 3: Normalized curvature $\bar{\kappa}=\kappa h$ versus oil fraction $f$ for a naturally straight beam and a naturally curved beam with silicone oil in the outer layer. The blue solid lines represent the 1D reduced model; the red triangles represent the experimental results.

We assume that $\lambda_{o i}$ and $\lambda_{o e}$ are determined by the bulk contraction due to oil extraction from the bilayer PDMS beam. Specifically, as oil is only present in the external layer, we set $\lambda_{o i}=1$, that is, we consider the inner layer as a passive layer. On the other hand, we assume that oil is completely extracted from the external layer and set

$$
\lambda_{o e}=(1-f)^{1 / 3} \quad \text { with } \quad f=V_{o} /\left(V_{p}+V_{c-l}+V_{o}\right),
$$

with the oil fraction $f=x /(11+x)$ defined as the ratio between the amount of oil $V_{o}$ and the total volume of the layer containing the oil, that is, the sum of the volume $V_{p}$ of the prepolymer, the volume $V_{c-l}$ of the cross-linker and $V_{o}$. It holds: $f<1$ and $\lambda_{o e}<1$. In our experiments, $\alpha \simeq 1, \beta=1 / 2, \Gamma=\lambda_{o e}=(1-f)^{1 / 3}$. Moreover, for small initial curvatures $\bar{\kappa}_{c} \rightarrow 0$ we get a simpler formula from equation 3.1 which is valid for almost flat beams:

$$
\kappa^{\mathrm{flat}}=\frac{12 \lambda_{o e}\left(1-\lambda_{o e}\right)\left(\lambda_{o e}+1\right)^{2}\left(\lambda_{o e}^{2}+6 \lambda_{o e}+1\right)}{h\left(\lambda_{o e}^{4}+3 \lambda_{o e}^{3}+8 \lambda_{o e}^{2}+3 \lambda_{o e}+1\right)^{2}} .
$$

Equations (3.1) and (3.3) deliver the curvature of the beam axis for a naturally curved and flat beam, respectively. Figure 3 shows that they are in excellent agreement with experimental data.

\section{Shape-shifting of cylindrical shells due to oil extraction}

The good agreement between experimental and explicit results for beams, suggested to study the deformations induced by oil extraction in bilayer PDMS cylindrical shells within the context of three-dimensional finite elasticity with distortions (natural deformations). These latter, as for the beams, are induced by oil extraction from the outer layer. The difference between curved beams and cylindrical shell is in the aspect ratio: small aspect ratios identify beams, large aspect ratios identify cylindrical shells.

Similarly to what we did for the beam curvature formulas (3.1) and (3.3), we assume that 
a bulk contraction corresponds to the complete extraction of oil from the external layer; it determines the stationary configuration of the cylindrical shell we aim to characterize. The reference state $\mathcal{B}$ of the $3 \mathrm{D}$ continuum is a bilayer cylindrical shell with a principal curvature $\kappa_{2} \neq 0$ equal to the curvature $\kappa_{c}$ of the beam cutted from it in the hoop direction (and $\kappa_{1}=0$ ). Figure 4 shows the reference states of cylindrical shells (left top and center cartoons) and beam (left bottom cartoon). Moreover, denoted with $L$ the length of the beam, as well as the arc length of the cylindrical shells(beam), and with AR the ratio between the width $w$ of the cylindrical shells(beam) and $L$, it holds AR $=0.1$, $\mathrm{AR}=0.5$ and $\mathrm{AR}=1$ for the beam and the cylindrical shells in the left cartoons in figure 4.

The dynamics of the process is beyond the scope of the communication and we focus on the equilibrium states of the polymer bodies. Introduced as state variable of the problem the displacement field $\mathbf{u}$ from $\mathcal{B}$, we denote with $x=X+\mathbf{u}(X)$ the position of a point $X \in \mathcal{B}$ at the steady state attained after oil extraction. We multiplicatively decompose the deformation gradient $\mathbf{F}=\mathbf{I}+\nabla \mathbf{u}$ in a pure inelastic deformation (bulk contraction) $\mathbf{F}_{o}$ and an elastic deformation $\mathbf{F}_{e}$. We also assume that $\mathbf{F}_{o}=\lambda_{o} \mathbf{I}$ with $\lambda_{o}=1$ in the passive layer and $\lambda_{o}<1$ in the active layer. On the other hand, the elastic deformation $\mathbf{F}_{e}$ is assumed to be isochoric, that is, such that $\operatorname{det} \mathbf{F}_{e}=1$. Hence, volume changes are only due to the distortion $\lambda_{o}$ which models the bulk contraction in the active layer due to oil extraction. It can be shown 23 that the following constitutive equations hold for the (reference) stress $\mathbf{S}$, corresponding to an elastically incompressible neo-Hookean material:

$$
\mathbf{S}=G \lambda_{o} \mathbf{F}-p(\operatorname{det} \mathbf{F}) \mathbf{F}^{-T},
$$

with $p$ the reactive stress maintaining the volumetric constraint $\operatorname{det} \mathbf{F}_{e}=1.3$

We implement within the finite element code COMSOL Multiphysics the mechanical equilibrium equation $\operatorname{div} \mathbf{S}=\mathbf{0}$, in a weak form, together with the boundary conditions of zero tractions. Eventually, we assume some boundary conditions on $\mathbf{u}$ that eliminate any rigid motions without generating reaction forces. We look for the solutions of the stationary problem corresponding to different values of the deformation $\lambda_{o}$, and for different AR (keeping fixed the arc length $L$ ). We set $L=35 \mathrm{~mm}, h=4 \mathrm{~mm}, \beta=1 / 2$, $G_{i}=G_{e}=1 \mathrm{MPa}$ and fixed the initial curvature $\kappa_{2}=\kappa_{c}=50 \mathrm{~m}^{-1}$ of the cylinder.

Figure 4 shows the outcomes of the numerical simulations for AR $=0.1$ (bottom row of cartoons corresponding to a curved beam), $\mathrm{AR}=0.5$ and $\mathrm{AR}=1$ (center and top rows of cartoons, respectively, corresponding to a cylindrical shell). Bulk contraction of the external layer increases from left to right. It corresponds to a distortion $\lambda_{o}$ decreasing from left to right in the top, center and bottom rows of cartoons and taking the values $\lambda_{o}=(1,0.95,0.875,0.8,0.75)$. The corresponding values of the oil fraction are $f=(0,0.14,0.33,0.49,0.58)$. So, left top, center and bottom cartoons correspond to the reference states of beam and cylindrical shells. As expected, to decreasing values of $\lambda_{o}$, the bilayer beam undergoes curvature reduction, flattening and eversion. On the

\footnotetext{
${ }^{3}$ It is worth noting that, due to the elastic incompressibility constraint, $\operatorname{det} \mathbf{F}=\lambda_{o}^{3}$.
} 


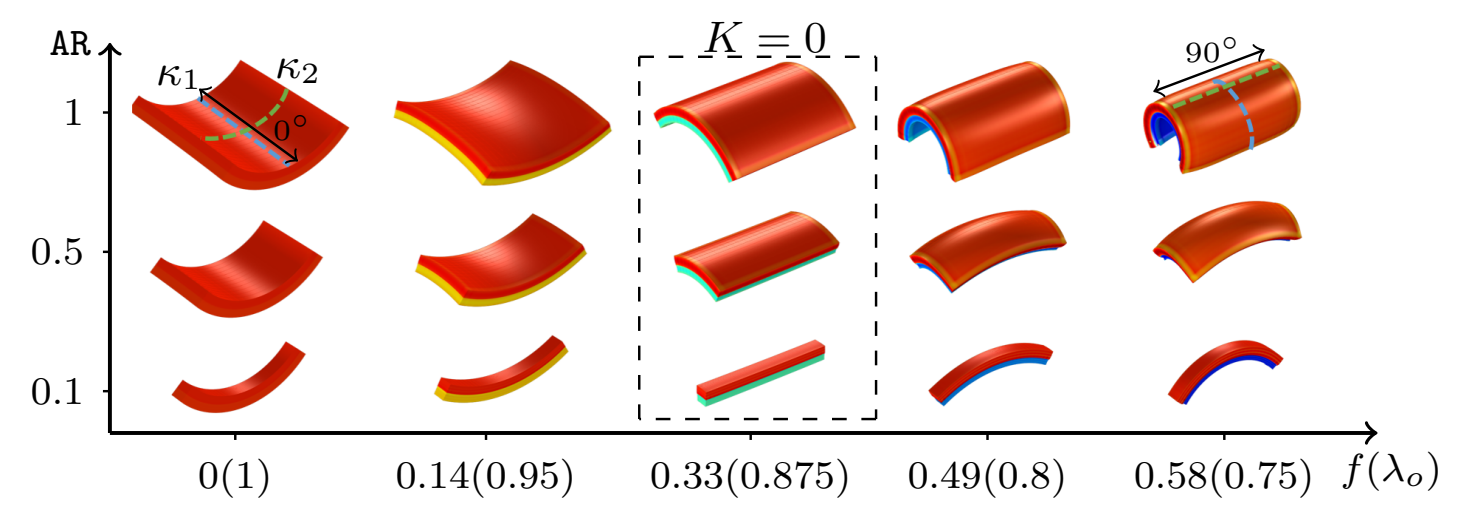

Figure 4: Morphing of bilayer naturally curved beam (bottom) and cylindrical shells (center and top) due to a bulk contraction $\lambda_{o}$ in the outer layer. The corresponding values of the oil fraction increase from left to right. The initial curvature $\kappa_{2}=\kappa_{c}$ can be find by looking at the $f=0$ column. At $\lambda_{o}=\lambda_{o}^{\star}=0.875\left(f=f^{\star}=0.33\right)$ (third column) the Gaussian curvature $K$ vanishes.

contrary, the morphing of the shells with $\mathrm{AR}=0.5$ and $\mathrm{AR}=1$ shown in figure 4 is noteworthy.

Firstly, at $\lambda_{o}=\lambda_{o}^{\star}=0.875$, corresponding to $f=f^{\star}=0.33$, we get cylindrical shells with the principal curvature axes switched with respect to the reference state: $\kappa_{1} \neq 0$ and $\operatorname{sign} \kappa_{1}=-\operatorname{sign} \kappa_{2}$ (see the cylindrical shells within the dashed frame in figure 4 . third column).

The value $\lambda_{o}^{\star}$ which identifies the switched cylinder is the same for any AR. Moreover, changing the initial curvature $\kappa_{2}$, the value $\lambda_{o}^{\star}$ changes even if it is still the same for any AR (see the inset in figure 5). Hence, given the initial curvature, we can evaluate the amount of oil to add to the polymer mixture to produce the shift of the two axes of the cylinder. For $\lambda_{o}^{\star}<\lambda_{o}<1$, the beam decreases its curvature and flattens, while the cylindrical shells morph into cylindrical shells with the axes of principal curvatures switched, passing through saddle-like shapes (figure 4, second and third columns from left). For $\lambda_{o}<\lambda_{o}^{\star}$, that is, $f>f^{\star}$, the beam everts, the shell with AR $=0.5$ attains a double-curved shape with $\kappa_{1}<0$ and $\kappa_{2}<0$, and the shell with $\mathrm{AR}=1$ bends along the direction orthogonal to the initial one (figure 4 , fourth and fifth column from left).

The morphing described above via the cartoons in figure 4 is also shown in figure 5 through the values taken by the normalized Gaussian curvature $\bar{K}=K h^{2}$, with $K=\kappa_{1} \kappa_{2}$, evaluated at the center of the middle surface in the stationary configuration at different values of $\lambda_{o}$ from 1 to 0.75 , for several AR ranging between 0.1 and 1 . For any AR, all the curves cross the zero line in two points: at $\lambda_{o}=1$, which corresponds to the reference state, and at $\lambda_{o}=\lambda_{o}^{\star}$, corresponding to the cylindrical shells with the axes of principal curvature switched with respect to the reference axes. Interestingly, the value $\lambda_{o}=\lambda_{o}^{*}$ corresponds to the value which makes null the curvature of a naturally curved beam (see equation (3.1)).

Figure 5 also shows as $\lambda_{o}^{\star}$ identifies the transition from saddle-like shapes and shapes with positive Gaussian curvature. Indeed, it can be observed that for $\lambda_{o}>\lambda_{o}^{*}$, we have 
$K<0$ for all the aspect ratios (saddle-like shape); for $\lambda_{o}<\lambda_{o}^{*}$, we have $K>0$ for $\mathrm{AR}=0.1,0.4,0.7$, and $K \simeq 0$ for $\mathrm{AR}=1$.

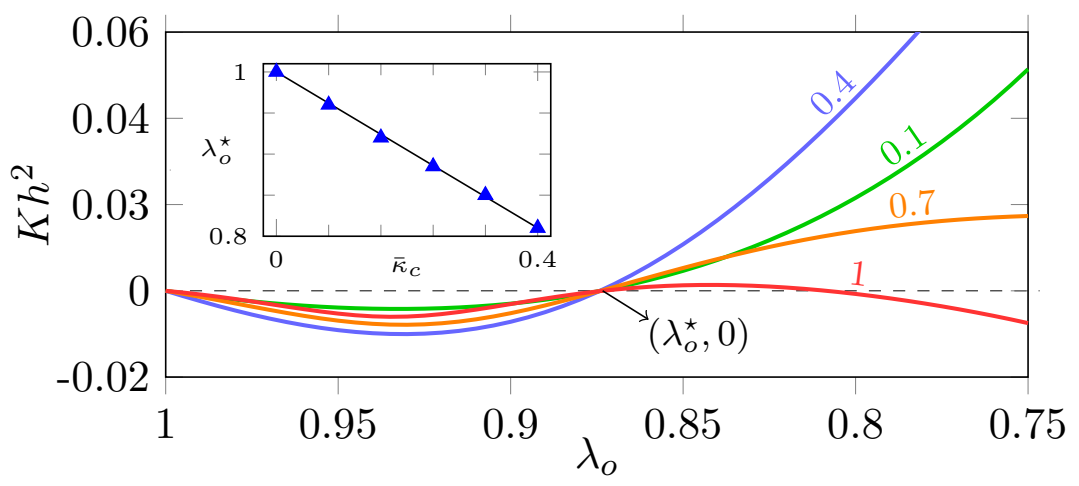

Figure 5: Normalized Gaussian curvature $\bar{K}=K h^{2}$ versus the bulk contraction $\lambda_{o}$ varying from 1 to 0.75 , for aspect ratios AR $=0.1,0.4,0.7,1$ (green, bleu, orange, red lines). The inset shows the dependence of the $\lambda_{o}^{\star}$ on the initial dimensionless curvature $\bar{\kappa}_{2}$ of the cylinder; the pattern corresponds to $L=35 \mathrm{~mm}$, $h=4 \mathrm{~mm}, \beta=1 / 2, G_{i}=G_{e}=1 \mathrm{MPa}$.

In figure6, we show how the transition from a given cylindrical shell to the cylindrical shell with switched axes changes when the normalized initial curvature $\bar{\kappa}_{c}=\kappa_{c} h$ of the shell decreases. The normalized principal curvatures $\kappa_{1} h$ (blue lines) and $\kappa_{2} h$ (orange lines) are evaluated at the center of the middle surface in the stationary configuration at different values of $\lambda_{o}$ from 1 to 0.85 , for $\bar{\kappa}_{c}=0.2,0.1,0.05$ and keeping fixed $\mathrm{AR}=1$. It is observed that the range of $\lambda_{o}$ values which allows to get a saddle-like shape is smaller and smaller as the cylindrical shell is thinner and thinner, and the green band in figure 6 is narrow and narrow. These observations allow us to make a precise design of the recipe used to realize the PDMS layer with oil inside, once fixed the thickness and the curvature of the cylinder. 




Figure 6: Normalized principal curvatures $\kappa_{1} h$ (blue lines) and $\kappa_{2} h$ (orange lines) versus the bulk contraction $\lambda_{o}$ varying from 1 to 0.85 , for $\bar{\kappa}_{c}=0.2$ (top plot), $\bar{\kappa}_{c}=0.1$ (center plot), $\bar{\kappa}_{c}=0.05$ (bottom plot), and keeping fixed $\mathrm{AR}=1$. The green area identifies, for each value of $\bar{\kappa}_{c}$, the range of $\lambda_{o}$ for which a saddle-like shape is attained.

\section{Conclusions}

We investigated the shape-shifting of bilayer polymer beams and cylindrical shells due to oil extraction. Polymer beams are made to evert through oil extraction from the outer layer. The morphing mechanism is also studied through an explicit model which accurately describes the observed morphing. The good agreement between experiments and modeling in beams inspired the computational study of cylindrical shells under the same working conditions, which will be supported in the next future by the appropriate experimental tests and also reviewed in view of the recent study presented in Ref. [24].

\section{Acknowledgments}

DB and PN would like to thank MIUR (Italian Minister for Education, Research, and University) and the PRIN 2017, Mathematics of active materials: From mechanobiology to smart devices, project n. 2017KL4EF3, for financial support.

\section{Conflicts of interest}

There are no conflicts to declare. 


\section{References}

[1] A. L. Thangawng, R. S. Ruoff, M. A. Swartz, M. R. Glucksberg, An ultra-thin pdms membrane as a bio/micro-nano interface: fabrication and characterization, Biomedical Microdevices 9 (4) (2007) 587-595. doi:10.1007/s10544-007-9070-6.

URL https://doi.org/10.1007/s10544-007-9070-6

[2] R. Mukhopadhyay, When pdms isn't the best Analytical Chemistry 79 (9) (2007) 3248-3253, pMID: 17523228. arXiv:https://doi.org/10.1021/ac071903e, doi:10.1021/ac071903e URL https://doi.org/10.1021/ac071903e

[3] J. N. Lee, C. Park, G. M. Whitesides, Solvent compatibility of poly(dimethylsiloxane)-based microfluidic devices Analytical Chemistry 75 (23) (2003) 6544-6554, pMID: 14640726. arXiv:https://doi.org/10. 1021/ac0346712 doi:10.1021/ac0346712

URL https://doi .org/10.1021/ac0346712

[4] X. Zhao, L. Li, B. Li, J. Zhang, A. Wang, Durable superhydrophobic/superoleophilic pdms sponges and their applications in selective oil absorption and in plugging oil leakages. J. Mater. Chem. A 2 (2014) 18281-18287. doi:10.1039/C4TA04406A

URL http://dx.doi.org/10.1039/C4TA04406A

[5] D. Zhu, S. Handschuh-Wang, X. Zhou, Recent progress in fabrication and application of polydimethylsiloxane sponges. J. Mater. Chem. A 5 (2017) 16467-16497. doi:10.1039/C7TA04577H URL http://dx.doi.org/10.1039/C7TA04577H

[6] A. Turco, E. Primiceri, M. Frigione, G. Maruccio, C. Malitesta, An innovative, fast and facile soft-template approach for the fabrication of porous pdms for oil-water separation J. Mater. Chem. A 5 (2017) 2378523793. doi: 10.1039/C7TA06840A

URL http://dx.doi.org/10.1039/C7TA06840A

[7] D.-X. Lu, Y.-L. Zhang, D.-D. Han, H. Wang, H. Xia, Q.-D. Chen, H. Ding, H.-B. Sun, Solvent-tunable pdms microlens fabricated by femtosecond laser direct writing, J. Mater. Chem. C 3 (01 2014). doi:10.1039/ C4TC02737 J

[8] B. Ji, M. Wang, Z. Guo, W. Hong, L. Wang, X. Wang, B. Yang, W. Wang, J. Liu, Wrinkled microelectrode interface based on oil-pretreated hyperelastic substrate, in: 2019 IEEE 32nd International Conference on Micro Electro Mechanical Systems (MEMS), 2019, pp. 561-564. doi:10.1109/MEMSYS.2019.8870820

[9] C. V. Rumens, M. A. Ziai, K. E. Belsey, J. C. Batchelor, S. J. Holder, Swelling of pdms networks in solvent vapours; applications for passive rfid wireless sensors. J. Mater. Chem. C 3 (2015) 10091-10098. doi:10.1039/C5TC01927C URL http://dx.doi.org/10.1039/C5TC01927C

[10] Y. Liu, H. Zhang, J. Zhang, Y. Zheng, Constitutive modeling for polymer hydrogels: A new perspective and applications to anisotropic hydrogels in free swelling European Journal of Mechanics - A/Solids 54 (2015) 171 - 186. doi:http://dx.doi.org/10.1016/j.euromechsol.2015.07.001. URL http://www.sciencedirect.com/science/article/pii/S0997753815000753

[11] D. H. Kim, M. C. Jung, S.-H. Cho, S. H. Kim, H.-Y. Kim, H. J. Lee, K. H. Oh, M.-W. Moon, Uv-responsive nano-sponge for oil absorption and desorption Scientific Reports 5 (12908) (2015). doi:10.1038/srep12908 URL https ://doi .org/10.1038/srep12908

[12] D. C. Stanier, J. Ciambella, S. S. Rahatekar, Fabrication and characterisation of short fibre reinforced elastomer composites for bending and twisting magnetic actuation, Composites Part A: Applied Science and Manufacturing 91 (2016) 168 -176. doi:https://doi.org/10.1016/j.compositesa.2016.10.001 URL http://www.sciencedirect.com/science/article/pii/S1359835X16303281

[13] R. Dangla, F. Gallaire, C. N. Baroud, Microchannel deformations due to solvent-induced pdms swelling Lab Chip 10 (2010) 2972-2978. doi:10.1039/C003504A

URL http://dx.doi.org/10.1039/C003504A 
[14] A. Hourlier-Fargette, J. Dervaux, A. Antkowiak, S. Neukirch, Extraction of silicone uncrosslinked chains at air-water-polydimethylsiloxane triple lines, Langmuir 34 (41) (2018) 12244-12250, pMID: 30199255. arXiv: https://doi.org/10.1021/acs.langmuir.8b02128 doi:10.1021/acs.langmuir.8b02128 URL https://doi.org/10.1021/acs .langmuir.8b02128

[15] B. Liu, J. Fu, Modulating surface stiffness of polydimethylsiloxane (pdms) with kiloelectronvolt ion patterning, Journal of Micromechanics and Microengineering 25 (06 2015). doi:10.1088/0960-1317/25/6/065006

[16] J. Xie, X. Han, H. Ji, J. Wang, J. Zhao, C. Lu, Self-supported crack-free conducting polymer films with stabilized wrinkling patterns and their applications Scientific Reports 6 (36686) (2016). doi:10.1038/ srep36686

URL https://doi.org/10.1038/srep36686

[17] J. Jackson, A. Chen, H. Zhang, H. Burt, M. Chiao, Design and near-infrared actuation of a gold nanorod-polymer microelectromechanical device for on-demand drug delivery, Micromachines 9 (1) (2018). doi: $10.3390 / \mathrm{mi} 9010028$

URL https://www .mdpi.com/2072-666X/9/1/28

[18] L. Chia, P. Bollgruen, A. Egunov, D. Mager, F. Malloggi, J. Korvink, V. Luchnikov, Vapour processed selfrolled poly(dimethylsiloxane) microcapillaries form microfluidic devices with engineered inner surface, Lab on a chip 13 (08 2013). doi:10.1039/c3lc50542a

[19] T. Sigaeva, R. Mangan, L. Vergori, M. Destrade, L. Sudak, Wrinkles and creases in the bending, unbending and eversion of soft sectors Proceedings of the Royal Society A: Mathematical, Physical and Engineering Sciences 474 (2212) (2018) 20170827. arXiv:https://royalsocietypublishing.org/doi/pdf/10.1098/ rspa.2017.0827. doi:10.1098/rspa.2017.0827

URL https://royalsocietypublishing.org/doi/abs/10.1098/rspa.2017.0827

[20] D. Battista, M. Curatolo, P. Nardinocchi, Swelling-induced eversion and flattening in naturally curved gel beams International Journal of Mechanical Sciences 161-162 (2019) 105071. doi:https://doi.org/10.1016/ j.ijmecsci.2019.105071

URL http://www.sciencedirect.com/science/article/pii/S0020740319315954

[21] A. I. Egunov, J. G. Korvink, V. A. Luchnikov, Polydimethylsiloxane bilayer films with an embedded spontaneous curvature Soft Matter 12 (2016) 45-52. doi:10.1039/C5SM01139F

URL http://dx.doi .org/10.1039/C5SM01139F

[22] S. Timoshenko, Analysis of bimetal thermostats, JOSA 11 (1925) 233-255.

[23] M. Curatolo, S. Gabriele, L. Teresi, Swelling and growth: a constitutive framework for active solids Meccanica 52 (14) (2017) 3443-3456. doi:10.1007/s11012-017-0629-x URL https://doi.org/10.1007/s11012-017-0629-x

[24] D. Battista, M. Curatolo, P. Nardinocchi, Enforcing shaping of thin gel sheets by anisotropic swelling Mechanics of Materials 139 (2019) 103199. doi:https://doi.org/10.1016/j.mechmat.2019.103199 URL http://www.sciencedirect.com/science/article/pii/S016766361930599X 